REGARDS

SUR L'ECONOMIE ALLEMANDE

BULLETIN ECONOMIQUE DU CRAC

\section{Regards sur l'économie allemande}

Bulletin économique du CIRAC

$66 \mid 2004$

Varia

\title{
L'enjeu de la pile à combustible et ses réseaux en
}

Allemagne

Isabelle Bourgeois

\section{(2) OpenEdition}

1 Journals

Édition électronique

URL : http://journals.openedition.org/rea/3858

DOI : $10.4000 /$ rea.3858

ISBN : 978-2-8218-0828-7

ISSN : 1965-0787

Éditeur

CIRAC

Édition imprimée

Date de publication : 1 mai 2004

Pagination : 25-32

ISSN : 1156-8992

Référence électronique

Isabelle Bourgeois, «L'enjeu de la pile à combustible et ses réseaux en Allemagne », Regards sur l'économie allemande [En ligne], 66 | mai 2004, mis en ligne le 13 octobre 2009, consulté le 14 novembre 2019. URL : http://journals.openedition.org/rea/3858 ; DOI : 10.4000/rea.3858 


\section{L'enjeu de la pile à combustible et ses réseaux en Allemagne}

\section{Isabelle Bourgeois}

20 équipes de collégiens et lycéens de Rhénanie du Nord-Westphalie participent en ce moment à un concours dont l'enjeu est l'alimentation électrique d'un téléphone portable grâce à une pile à combustible. Sélectionnées parmi 174 équipes candidates, elles se sont vu remettre en février par la ministre de la Recherche du Land chacune une pile à combustible en kit offerte par le groupe Fuel Cell Europe (Hambourg) et un portable fourni par Vodafone D2 (succursale de Hattingen). Les équipes lauréates gagneront un « HyRunner », modèle réduit de voiture à hydrogène produit par la société h-tec Wasserstoff-Energie-Systeme GmbH (Lübeck). A l'échelle nationale, un Prix de l'innovation pour l'hydrogène et la pile à combustible, décerné par la Fédération allemande de l'hydrogène DWV, récompensera les meilleurs thèses et travaux de fin d'étude réalisés courant 2004. L'intérêt pour ces technologies est très vif outre-Rhin, où le savoir-faire industriel est diffusé auprès d'une très large opinion avec l'appui des pouvoirs publics.

Sources 'propres' d'électricité et de chaleur, les technologies de l'hydrogène intéressent vivement une opinion allemande très soucieuse de préserver l'environnement. Ne produisant que peu de $\mathrm{CO}_{2}$, elles présentent en outre l'avantage de permettre la diversification des sources d'énergie primaire et d'optimiser en particulier les énergies renouvelables. Intéressant à ce titre en premier lieu les ministères de l'environnement, elles trouvent tout aussi naturellement leur place dans l'Energiemix allemand (voir REA 65/04), puisqu'elles sont à mêmes de réduire à terme la dépendance énergétique : l'hydrogène, vecteur omniprésent et inépuisable, pourrait relayer progressivement les énergies fossiles. Pierre angulaire de la future " économie de l'hydrogène " vers laquelle tendent aujourd'hui les politiques d'innovation de l'UE et, plus généralement de tous les pays industrialisés, la pile à combustible commence à devenir aussi un enjeu stratégique de la politique économique et industrielle allemande. Moins pour le potentiel 'révolutionnaire' qu'on lui prête en matière d'approvisionnement énergétique que pour son effet levier sur l'innovation et la compétitivité du pays.

\section{Quatre principaux types de piles à combustible}

Le principe de la pile à combustible a été découvert en 1839 par le Britannique William Grove : la réaction électrochimique de l'hydrogène avec de l'oxygène permet de produire de l'électricité directement. La réaction s'effectue grâce à deux électrodes séparées par un électrolyte. Selon les caractéristiques de ces éléments, le mode de production d'électricité varie, de même que le niveau de température dégagé. Les applications (portable, transports, stationnaire) varient en conséquence selon les quatre principaux types de piles à combustible existants :

- la pile à membrane échangeuse de protons, ou Proton Exchange Membrane Fuel Cell PEMFC, dont l'électrolyte est un polymère solide,

- la pile à carbonate fondu, ou Molten Carbonate Fuel Cell - MCFC (électrolyte : sels fondus),

- la pile à oxyde solide, ou Solid Oxide Fuel Cell - SOFC (électrolyte : céramique),

- la pile à méthanol direct, ou Direct Methanol Fuel Cell - DMCF (électrolyte : polymère).

Les premières piles à combustible ont été construites dans les années 1960 pour les capsules Gemini. Ces applications spatiales ont ensuite déclenché les activités de R\&D industrielle dans le domaine d'un nouveau type d'approvisionnement énergétique à partir des années 1980. Depuis, les technologies liées à la pile sont dans l'ensemble maîtrisées. II n'en va pas de même de son combustible: l'hydrogène, qui n'existe pas naturellement à l'état pur, et dont différents modes de production sont en cours de développement. Les pays industrialisés et l'UE viennent de lancer une vaste politique de soutien à la maîtrise de ce vecteur énergétique. 
Pile à combustible : des enjeux multiples

Les domaines d'application de la pile à combustible sont de trois ordres : l'alimentation en énergie d'appareils portables, la propulsion ou l'alimentation électrique des moyens de transport, ainsi que la production stationnaire de chaleur et d'électricité. L'avantage de l'hydrogène utilisé comme combustible est la facilité de son stockage ; il devrait donc s'imposer dans tous les domaines où un approvisionnement continu en électricité est requis, mais difficile à réaliser. La pile à combustible devrait donc se substituer largement aux batteries traditionnelles et ouvrir de nouvelles perspectives à la génération mobile d'électricité dans le secteur des transports. Parallèlement, elle permettra une production décentralisée d'électricité et/ou de chaleur, ce qui en fait un enjeu primordial dans le contexte de la libéralisation des marchés énergétiques, mais aussi de la diversification des sources d'approvisionnement.

\section{Les principales applications des piles à combustible}

\begin{tabular}{|c|c|c|c|c|c|c|}
\hline \multirow[t]{3}{*}{ Applications } & \multicolumn{2}{|c|}{ Portable } & \multicolumn{2}{|c|}{ Transports } & \multicolumn{2}{|c|}{ Stationnaire } \\
\hline & micro & $\operatorname{mini}$ & compacte & propulstion & $\begin{array}{c}\text { énergie } \\
\text { domestique }\end{array}$ & $\begin{array}{c}\text { énergie } \\
\text { décentralisée }\end{array}$ \\
\hline & $\begin{array}{c}\text { Télé- } \\
\text { phones } \\
\text { portables }\end{array}$ & $\begin{array}{l}\text { Ordinateurs } \\
\text { portables, } \\
\text { outillage } \\
\text { électrique }\end{array}$ & $\begin{array}{l}\text { cycles, } \\
\text { APU *) }\end{array}$ & $\begin{array}{l}\text { Véhicules } \\
\text { particuliers et } \\
\text { industriels, } \\
\text { générateurs } \\
\text { électriques }\end{array}$ & $\begin{array}{c}\text { Habitat } \\
\text { individuel }\end{array}$ & $\begin{array}{l}\text { Industrie, } \\
\text { approvisionne- } \\
\text { ment urbain }\end{array}$ \\
\hline Types de piles & $\begin{array}{l}\text { PEMFC, } \\
\text { DMFC }\end{array}$ & $\begin{array}{l}\text { PEMFC, DMFC, } \\
\text { SOFC (APU) }\end{array}$ & PEMFC & $\begin{array}{l}\text { PEMFC, } \\
\text { SOFC }\end{array}$ & $\begin{array}{l}\text { PEMFC, } \\
\text { MCFC, } \\
\text { SOFC }\end{array}$ & PEMFC, DMFC \\
\hline Combustible & $\begin{array}{l}\text { Hydro- } \\
\text { gène, } \\
\text { méthanol }\end{array}$ & $\begin{array}{c}\text { Hydrogène, } \\
\text { méthanol, } \\
\text { diesel, essence }\end{array}$ & $\begin{array}{c}\text { Gaz naturel, gaz } \\
\text { liquide, fuel } \\
\text { domestique, } \\
\text { hydrogène, } \\
\text { biomasse }\end{array}$ & $\begin{array}{l}\text { Hydrogène, } \\
\text { méthanol }\end{array}$ & $\begin{array}{l}\text { Hydrogène, } \\
\text { méthanol, } \\
\text { diesel, } \\
\text { essence }\end{array}$ & $\begin{array}{c}\text { Gaz naturel, gaz } \\
\text { liquide, fuel } \\
\text { domestique, } \\
\text { hydrogène, } \\
\text { biomasse }\end{array}$ \\
\hline
\end{tabular}

Source des données : L-B-Systemtechnik, Brennstoffzellen- und Wasserstoff-Technologien als wirtschaftliche Chance für Hamburg, 2004. * APU: générateur électrique auxiliaire (Auxiliary Power Unit) ; **) au plus tôt.

Une Plateforme européenne pour les technologies de l'hydrogène
Ses membres allemands sont représentatifs
Les technologies liées à la pile à combustible ont dès lors un effet levier transversal non négligeable sur la structuration à la fois de la filière énergétique et, plus généralement, de tous les produits et activités impliquant une alimentation électrique - on compare souvent leur impact potentiel à celui des semiconducteurs. Pour l'instant, le calendrier de la commercialisation prévisionnelle des divers types de piles varie beaucoup : si le savoir-faire industriel en matière de piles est largement développé, le recours à l'hydrogène soulève des questions de sécurité, de coût et d'infrastructures encore inégalement maîtrisées. Ces questions, qui ne sont pas spécifiques à l'Allemagne, avaient motivé le lancement par la Commission européenne, en janvier 2004, d'une « Plateforme européenne pour les technologies de l'hydrogène et des piles à combustible » destinée à renforcer la compétence de l'UE dans « l'économie de l'hydrogène » et lui permettre de contribuer au partenariat international conclu en novembre 2003 avec les Etats-Unis, l'Inde, la Chine et douze autres pays.

Cette plateforme européenne est pilotée par un comité consultatif composé des acteurs clefs du secteur européen de l'hydrogène. Parmi ses 35 membres figurent 10 représentants allemands (mais seulement trois français et trois britanniques, parmi lesquels le président). Leur recrutement reflète la structuration de la filière hydrogène outre-Rhin, traduisant également le haut degré de globalisation des activités dans ce domaine. II révèle, par delà, le fonctionnement en réseaux de partenariat, caractéristique de l'industrie allemande en général, et de l'innovation industrielle en particulier. Pile à combustible et hydrogène ne font pas exception au schéma national d'innovation allemand, caractérisé par une étroite coopération des réseaux d'innovation et de partenariat entre les principales branches industrielles concernées, ainsi que par un flux permanent de transfert de savoirs entre acteurs privés, publics, et l'opinion. 


\section{Plateforme européenne pour l'hydrogène et les piles à combustible. Une participation allemande représentative.}

Parmi les membres allemands de la plateforme, on trouve, bien sûr, l'un des deux représentants des grandes ONG spécialisées dans la protection de l'environnement: WWF (l'autre, Greenpeace, y siège au titre des Pays-Bas), ce qui reflète les préoccupations environnementales prononcées des Européens du Nord. On y trouve également un représentant des pouvoirs publics, délégué en l'occurrence par le Land de Rhénanie du Nord-Westphalie, principal site énergétique et industriel allemand. Y figure ensuite le représentant d'une fondation associant acteurs publics (Land de Bade-Wurtemberg, Universités de Stuttgart et d'Ulm, Deutsches Zentrum für Luft- und Raumfahrt) et privés (dont EnBW, Bosch, DaimlerChrysler ou Telefunken Electronic). Cette fondation Zentrum für Sonnenenergie- und Wasserstoff-Forschung (ZSW, Stuttgart), spécialisée dans la recherche sur l'énergie solaire et l'hydrogène, était née en 1988 avec pour mission de soutenir la recherche fondamentale et appliquée dans ces domaines, ainsi que ses applications industrielles, et de diffuser l'information sur ces innovations. Sa présence dans la plateforme reflète le rôle fondamental des fondations dans le financement de la R\&D allemande (voir REA 57/02). Quant aux fédérations professionnelles, acteurs clefs de la diffusion du savoir et du transfert technologique outre-Rhin, elles sont également présentes en la personne d'un membre du directoire de la régie des transports publics de Cologne, affiliée à l'association internationale des opérateurs de transports urbains et régionaux UITP.

Les six autres membres allemands de la plateforme européenne se recrutent parmi les grands acteurs industriels de la filière hydrogène : Linde AG (gaz, élévateurs industriels, technologies du froid), Siemens AG (génération d'énergie notamment), Ballard Power Systems AG (filiale allemande du groupe canadien, leader mondial des piles à combustible dans les transports automobiles), Umicore AG \& Co. KG (filiale allemande du groupe belge de métaux et matériaux, spécialiste notamment des membranes pour piles à combustible), ainsi que DaimlerChrysler et BMW (acteurs historiques des véhicules à hydrogène). La composition de cette plateforme est représentative du paysage allemand de la pile à combustible et de l'hydrogène.

Le savoir faire allemand en matière de pile à combustible est inégalement développé, reflétant la diversité de l'état mondial d'avancement de la R\&D dans les multiples domaines concernés. Le segment actuellement le plus proche de la commercialisation est celui des applications mobiles (ordinateurs ou téléphones portables) où la génération d'énergie peut se faire à l'aide de cartouches d'hydrogène, sur le modèle des cartouches de gaz utilisés en camping. L'approvisionnement en hydrogène ne pose aucun problème majeur, ce gaz étant disponible en quantité largement suffisante pour ce type d'application - en tant que produit dérivé issu de l'électrolyse et, surtout, du reformage (gaz naturel, méthanol). En outre, infrastructures et logistique d'approvisionnement existent (commerce de détail, stations service) ou peuvent rapidement être mises en place. Le marché des portables à hydrogène a un fort potentiel étant donné la faiblesse actuelle des batteries au lithium en comparaison de la consommation électrique croissante de ces équipements.

Les géants de l'informatique, mais aussi les développeurs de piles comme la nanostock plasturgiste Masterflex AG (Gelsenkirchen), la start up Smart Fuel Cell AG (SFC, Munich) ou la jeune P21 GmbH (créée en 2001 près de Munich), prévoient de lancer la production de série courant 2004, après la présentation de leur produits lors des salons CeBIT, H2/FC de Hanovre ou H2Expo de Hambourg. Masterflex avait développé sa pile (une PEMFC périphérique) avec le Fraunhofer Institut für Solare Energiesysteme (ISE, Fribourg), centre de recherche appliquée pilote en la matière. P21, une spin off de Mannesmann/ Vodafone née lors de leur fusion, dispose de son propre savoir-faire en matière de PEMFC pour téléphones portables; elle a une quarantaine de brevets à son actif. Quant à la société SFC, elle développe des piles au méthanol (DMFC) intégrées dans les portables. Plus miniaturisées que les PEMFC, les DMFC semblent avoir la faveur des observateurs. Mais dans les deux cas, la commercialisation à grande échelle se heurte encore au prix de ces innovations.

Dans le domaine des transports, l'état des lieux est plus complexe. Les constructeurs automobiles allemands sont en pointe en Europe, depuis que DaimlerChrysler a présenté en 1994 le premier véhicule à hydrogène au monde : la NECAR 1. Aujourd'hui, grâce au partenariat avec le canadien Ballard Power Systems, la pile a été réduite en volume, sa puissance ayant été décuplée dans
Bientôt sur le marché : les applications portables

DMFC contre PEMFC

Transports : l'automobile allemande en pointe en Europe 
Commercialisation pas avant 2010 , par manque d'infrastructures

Véhicules industriels lourds : divers tests en cours

Transports urbains : DaimlerChrysler en pointe

Aéronautique : Airbus (Hambourg) contre Boeing (Madrid) le même temps. La NECAR 5, présentée en 2000, est équipée d'une pile au méthanol, ce qui la rend théoriquement commercialisable assez rapidement. 60 de ces véhicules, des Mercedes de Classe A, sont actuellement testés outreRhin. Dans le domaine des véhicules industriels légers, DaimlerChrysler coopère avec l'intégrateur UPS pour tester sur le marché américain un modèle Sprinter à hydrogène. Le groupe Ford, dont le siège européen est à Cologne, a présenté récemment la Focus FCEV Hybrid, dont cinq exemplaires sont aujourd'hui testés à Berlin dans le cadre d'un partenariat entre constructeurs automobiles (BMW, DaimlerChrysler, GM/Opel, MAN, VW), énergéticiens ou pétroliers (ARAL, BP, RWE, Shell et Total) et le ministère fédéral des Transports. Cette coopération germano-américaine, dédiée à une politique énergétique durable, vise à prendre de vitesse les concurrents japonais (Toyota et Honda).

La commercialisation de ces véhicules se heurte encore à une problème de poids ou de longévité de la pile, ainsi que de coûts: les membranes utilisées dans les piles sont recouvertes de platine. Selon le groupe Ballard, qui est aussi partenaire de Ford, les premiers véhicules particuliers à hydrogène ne seront commercialisés qu'après 2010. Le principal frein réside en effet dans l'absence d'infrastructures d'approvisionnement: pour équiper 1000 stations service (on en compte 16000 outre-Rhin), il faudrait investir 100 milliards $€$, estime la Fédération des ingénieurs VDI. Le second objectif de cette coopération germanoaméricaine est donc le développement du réseau d'infrastructures.

En matière de véhicules industriels lourds, un marché pourrait émerger plus tôt, étant donné que les autobus, élévateurs ou excavatrices ont une mobilité réduite en comparaison et, surtout, peuvent s'alimenter régulièrement dans les entrepôts ou sur le site industriel. A l'inverse des voitures particulières ou des camions, le poids de la pile à combustible est également un avantage dans ce segment, puisqu'il sert la stabilité des véhicules. Ainsi, le constructeur d'élévateurs et excavatrices Still $\mathrm{GmbH}$ (Hambourg), a équipé d'une pile à combustible un élévateur testé sur l'aéroport de Munich dans le cadre du projet ArgeMUC, lancé en 1997 et rebaptisé depuis H2MUC. Parmi les membres de ce groupe de travail, créé expressément par l'industrie pour affronter la concurrence des USA et du Japon, figurent ARAL, BMW, IAW, MAN, Linde, Neoplan ou Siemens. Bénéficiant du soutien du TÜV (contrôle technique et de sécurité), le projet est financé à parité par les entreprises et le ministère de l'Economie de Bavière (budget total : environ 16 millions $€$ ). D'autres utilitaires sont testés également, mais le pivot du projet est la production d'hydrogène sur le site et le ravitaillement automatique de la flotte ; les infrastructures sont également ouvertes aux particuliers, ce qui leur confère une valeur de 'produit d'appel'.

Dans le domaine des transports urbains, DaimlerChrysler teste depuis septembre 2003 trois autobus de type Citero sur le réseau de Hambourg, suivis de 27 autres à Stuttgart et huit autres villes européennes dans le cadre principalement du projet CUTE (Clean Urban Transport for Europe) de l'UE. Les partenaires sur le site pilote de Hambourg sont, outre la compagnie des transports urbains, le constructeur d'autocars Evobus, BP et l'électricien HEW. Le ravitaillement des bus en hydrogène d'origine éolienne s'effectue dans l'entrepôt. Si ce type de véhicules présente de nombreux avantages théoriques (silence, absence d'émissions), surtout en milieu urbain, leur coût prohibitif (1,2 million $€$ l'unité) ne laisse envisager qu'une commercialisation à long terme, d'autant que leur faible autonomie (200 km au maximum) renvoie à la question des infrastructures d'approvisionnement en hydrogène. Cette question est cruciale pour l'ensemble du secteur des transports civils dès lors que la pile à combustible y est développée en tant que moyen de propulsion.

Il n'en va pas de même lorsque la pile est conçue comme un générateur électrique auxiliaire (auxiliary power unit : $A P U$ ). C'est le cas de l'automobile, où les APU sont le second axe de R\&D poursuivis par les constructeurs de voitures particulières - les premiers modèles haut de gamme seront prochainement 
équipés pour alimenter l'électronique de bord -, ainsi que de véhicules industriels destinés au transport routier et qui, du fait de l'évolution de la logistique, sont de gros consommateurs d'électricité. C'est le cas également de l'aéronautique où la R\&D civile se concentre surtout sur les APU pour optimiser les flux électriques, hydrauliques, etc. Dans la course mondiale à l'innovation (APU et propulsion), Boeing et Airbus se livrent une concurrence acharnée sur le sol européen : alors que Boeing a choisi Madrid pour centre de développement, la R\&D d'Airbus est pilotée par le site de Hambourg. Quant à la MTU Aero Engines (Munich), leader mondial de la maintenance aéronautique racheté en janvier 2004 à DaimlerChrysler par la société américaine de private equity KKR, elle développe la pile à combustible selon divers axes, dont l'hydrogène liquide, dans le cadre notamment du programme international Cryoplane.

Les transports ferroviaires s'intéressent également de près à la pile à combustible. Une initiative était née à la fin des années 90 à Hambourg pour développer des SOFC de 500 à $6000 \mathrm{~kW}$ destinées à équiper notamment des locomotives. Elle associait industrie et recherche universitaire, mais n'avait pas pu trouver alors le soutien du ministère fédéral de la Recherche et des Technologies. Entre-temps, les USA ont lancé un programme militaire voisin, et l'Europe a découvert "l'économie de l'hydrogène ". Dès lors, toutes les applications liées au rail commencent à intéresser les pouvoirs publics outre-Rhin, d'autant que les transports urbains (comme les tramways, très répandus) sont potentiellement un domaine où la pile à combustible présente des avantages décisifs : l'absence d'émissions ou la suppression des caténaires et donc la réduction des problèmes liés aux fréquences électromagnétiques.

Les transports maritimes sont un domaine où la pile à combustible commence à être commercialisée, du moins ses applications militaires. La marine a ainsi commandé une série de sous-marins U31 équipés de PEMFC à la HDW Fuel Cell Systems GmbH (HFCS, Kiel). Dans ce domaine, la pile à combustible (propulsion et APU) tend à devenir un standard puisqu'elle permet de réduire la signature (sous-marins furtifs) et d'assurer la génération d'électricité et d'eau à bord. D'une manière générale, comme pour l'aviation ou les transports routiers, les applications APU devraient se généraliser rapidement pour couvrir les besoins liés à la montée en puissance de l'électronique de bord - dans les domaines militaire et civil. La société HFCS, par exemple, dont la PEMFC a été développée par Siemens, la Germanische Lloyd Certification GmbH (Hambourg) se chargeant du contrôle sécurité et de la certification, se concentre actuellement sur la navigation de plaisance où divers tests sont en cours (APU et reformage du diesel). Mais dans la marine civile, les questions centrales demeurent celles du type de pile et du combustible le plus adapté comme des infrastructures afférentes. Et pour les chantiers navals allemands qui, à l'instar de l'automobile, travaillent en étroite coopération avec les PME sous-traitantes, la mobilisation de ce réseau est aujourd'hui une priorité, en termes d'innovation, de compétitivité face au Japon, et de développement structurel régional.

Troisième domaine d'application: la génération stationnaire et décentralisée d'électricité - et souvent aussi de chaleur. Elle intéresse l'Allemagne à plusieurs égards: outre qu'elle intervient dans les question liées à l'épuisement progressif des énergies fossiles ou à l'objectif de la réduction des émissions de gaz à effet de serre, elle offre une perspective dans le délicat équilibre à trouver en matière de choix énergétiques. L'abandon du nucléaire se solde inévitablement par un recours accru aux énergies fossiles ou renouvelables. Or ces dernières ne couvrent encore que près de $8 \%$ de la consommation totale d'électricité, alors que l'Allemagne s'est engagée à porter leur part à $21 \%$ en 2010 (voir REA 65/04). Dès lors, les deux tiers de la centaine d'énergéticiens allemands (des électriciens aux régies communales) sont impliqués dans la R\&D.

L'enjeu politique est moins celui de la décentralisation de la production d'énergie (elle l'est depuis toujours outre-Rhin) que celui de la sécurisation et de la
Rail : un intérêt naissant

Marine : sous-marins furtifs et navigation de plaisance

Génération stationnaire d'énergie

Le marché visé :

I'habitat individuel 
Initiative Brennstoffzelle : une plateforme ad hoc ...

... reliée au programme Virtua Fuel Cell Power Plant de I'UE régulation de l'approvisionnement domestique. Pour les opérateurs, les enjeux sont industriels : de nombreuses centrales arrivant en fin de vie d'ici une dizaine d'années, la pile semble une alternative intéressante au renouvellement des capacités. Ainsi, Siemens Power Generation met actuellement en service une SOFC (production combinée chaleur/électricité) à Hanovre pour le compte d'E.ON Energie AG et de la régie municipale Stadtwerke Hannover AG. De leur côté, RWE Fuel Cells et MTU ont conclu en 2003 un joint venture pour la commercialisation vers 2005 d'une pile MCFC. Pour les gaziers, c'est un axe bienvenu de diversification, l'une des principales sources de production d'hydrogène étant le gaz. Les enjeux sont également commerciaux : $5 \%$ des 13,5 millions de maisons individuelles ou en copropriété que compte l'Allemagne pourraient être équipées en 2020, selon le cabinet Arthur D. Little. C'est dès lors sur l'habitat individuel et les PME que se concentrent les espoirs de voir se constituer à long terme un marché de la pile à combustible. Car les industriels, largement autonomes pour la production d'énergie, semblent privilégier d'autres solutions, à de rares exceptions près : ainsi Michelin, qui a équipé son site de production de Karlsruhe d'un « Hot Module » $(250$ kW) produit par MTU CFC Solutions.

Pour éviter l'éparpillement de la R\&D et créer des synergies dans les savoirfaire afin d'optimiser la commercialisation future de la pile à combustible, les énergéticiens EWE AG (Oldenburg), MVV Energie AG (Mannheim), Ruhrgas AG (Essen) et VNG (Leipzig) ont constitué en octobre 2002 une plateforme ad hoc: Initiative Brennstoffzelle (IBZ). Elle associe des partenaires institutionnels: l'Université de Duisburg, la Fédération des artisans du sanitaire, du chauffage et de la climatisation (Zentralverband Sanitär Heizung Klima, ZVSHK), l'Association scientifique et technique des filières de l'eau et du gaz (Deutsche Vereinigung des Gas- und Wasserfachs, DVGW) qui est l'instance d'auto-régulation de la branche en matière de sécurité et de spécification, ainsi que la Deutsche Energie Agentur GmbH (dena) qui se définit comme un " centre de compétence pour l'efficience énergétique » et dont les actionnaires sont l'Etat fédéral et la banque publique KfW (pour moitié chacun). Parmi les industriels associés figurent : le sidérurgiste et chauffagiste Buderus AG (Wetzlar, une filiale du groupe Bosch), ainsi que les chauffagistes allemands Vaillant AG (Remscheid) et Viessmann Werke GmbH \& Co. KG (Allendorf), et leur homologue suisse Sulzer Hexis AG (Winterthur).

L'IBZ a débouché sur deux projets pilotes, toujours en cours, où Sulzer Hexis AG et Vaillant AG testent sur le terrain rentabilité, fiabilité et longévité de leurs piles concurrentes. Le premier teste depuis 2001 sa HXS Premiere 1000, une pile à haute température (SOFC) destinée au chauffage central de maisons individuelles. Une seconde génération de piles sera mise à l'essai fin 2004 ou début 2005. De son côté, Vaillant AG teste depuis deux ans, dans l'habitat collectif et auprès des PME, la production combinée de chauffage et d'électricité, mettant en œuvre une PEMFC : une pile à basse température appelée EURO 1, et qui sera relayée prochainement par l'EURO 2. Lancés d'abord en Rhénanie du Nord-Westphalie, les essais se poursuivent depuis 2003 sur l'ensemble du territoire allemand, aux Pays-Bas, en Espagne et au Portugal dans le cadre du programme Virtual Fuel Cell Power Plant de I'UE. Sa finalité est d'expérimenter la mise en réseau des piles et leur raccordement au réseau électrique ; un poste de contrôle commande le système décentralisé de production d'électricité comme s'il s'agissait d'une centrale électrique 'classique' - mais celle-ci est virtuelle, d'où le nom du programme. Cette architecture impliquant la coopération des énergéticiens, les partenaires allemands de Vaillant AG sont EWE AG et Ruhrgas AG, ce dernier pilotant les tests outre-Rhin (coût total : 8,6 millions $€$, dont 3 millions $€$ financés par l'UE). Car pour les énergéticiens, la gestion de micro-réseaux de production isolés, mais reliés aux réseaux de distribution existants, est une expérience complexe et nouvelle. II faut développer de nouveaux concepts de distribution, mais aussi intégrer dans cette approche diversi- 
fiée les constructeurs de piles et leurs sous-traitants, ou l'artisanat pour la maintenance des installations.

Reste la question de la génération de l'hydrogène; car ce gaz très répandu n'existe pratiquement à l'état naturel que comme constituant d'autres corps. L'objectif, en Allemagne comme ailleurs, est de le produire à partir de sources renouvelables d'énergie primaire et de ramener les émissions de gaz à effet de serre à un niveau proche de zéro. Mais il s'agit pour l'instant d'un rêve lointain. Car pour l'instant, l'essentiel de la production mondiale d'hydrogène provient du raffinage du pétrole, une petite part seulement étant obtenue sous forme de dérivé par l'industrie chimique et une quantité négligeable étant générée par électrolyse de l'eau. Et les quantités produites aujourd'hui sont notoirement insuffisantes pour permettre l'émergence d'une « économie de l'hydrogène ".

L'industrie chimique allemande produit environ 1 milliard $\mathrm{m}^{3}$ d'hydrogène. Cela suffirait à couvrir la consommation annuelle de 1000 autobus municipaux, affirme une étude de la société L-B-Systemtechnik GmbH (LBST, Ottobrunn), membre du European Integrated Hydrogen Project (EIHP2). Or les clients de cet hydrogène sont des sociétés spécialisées comme Linde ou Air Liquide. L'extension de la clientèle se heurte principalement au coût de production. En comparaison, le reformage du gaz naturel (ou du méthanol produit à partir de gaz naturel, voire de biomasse) est actuellement la technique de génération d'hydrogène la plus avantageuse. Comme il s'agit d'un savoir-faire standard, le marché potentiel est énorme, le reformage pouvant s'effectuer dans tous types d'installations : centrale énergétique, station service, pile à combustible à usage domestique ou automobile... De nombreux industriels s'y intéressent, ainsi Caloric Anlagenbau $\mathrm{GmbH}$ (près de Munich, construction d'installations) ou CarboTech (Essen ; installations, forage, filtrage au charbon actif), la filière automobile dans son entier, de même que toutes les compagnies du gaz. Cette technique a néanmoins un inconvénient : elle produit du $\mathrm{CO}_{2}$. Dès lors, elle pourrait utilement être relayée à long terme par l'électrolyse de l'eau, préconise l'étude de la LBST ; le futur parc d'éoliennes offshore (20 GW d'ici 2020 environ) pourrait alors offrir une opportunité stratégique. D'ici 2018, les centrales nucléaires côtières seront mises hors service. Or la capacité de leurs réseaux d'interconnexion n'est que de $8 \mathrm{GW}$. Que faire de la surproduction d'électricité éolienne ? Elle pourrait être utilisée pour générer de l'hydrogène par électrolyse.

"L'économie de l'hydrogène » soulève un classique problème de la poule et de l'œuf. Dans de nombreux domaines, les piles à combustible sont sorties de la phase du prototype, et leur commercialisation pourrait être lancée à court ou moyen terme s'il y avait les infrastructures et l'approvisionnement nécessaires. Pour créer un marché de masse, il faut réduire le coût des diverses applications, mais il faut aussi assurer l'approvisionnement permettant l'émergence de ce marché de masse qui réduira in fine les coûts. Seuls l'action coordonnée de tous les acteurs impliqués et un soutien politique permettant d'apporter une solution à ce problème, l'UE a donc lancé récemment divers programmes de recherche et plateformes de coopération. Cette politique transversale, placée à la fois sous le signe du développement durable et de la compétitivité énergétique et industrielle de l'UE, se conjugue aux divers programmes de soutien à la recherche en matière de piles à combustibles menés par l'UE depuis 1989, ainsi qu'au programme Quick-Start lancé en 2003 dans le cadre de L'Initiative européenne pour la croissance et doté d'une enveloppe globale de 2,8 milliards $€$ de fonds publics et privés. Ces actions concernent la recherche, les transports, l'énergie, les infrastructures, l'industrie, l'environnement ou la formation.

Ajoutées aux divers axes de la politique d'innovation allemande, elles sont déclinées au plan national et régional en tant que partie intégrante des diverses politiques nationales de soutien à l'innovation : du soutien aux start ups et PME innovantes (ministères de l'économie) à la formation/recherche technologique (ministères de l'économie, de la recherche) en passant par les politiques de
Une production d'hydrogène largement insuffisante

2 sources possibles : reformage du gaz naturel, puis électrolyse

Actions coordonnées de l'UE...

... conjuguées en RFA aux politiques propres 
soutien au développement des énergies renouvelables (ministère de l'environnement), sans oublier les programmes de développement structurel régional, notamment dans les nouveaux Länder. C'est donc un complexe bouquet de politiques d'offre que proposent les pouvoirs publics outre-Rhin dans ce domaine comme dans d'autres (biotechnologies ou nanotechnologies, voir REA 57/02 et 64/03). Elles viennent en appui aux initiatives industrielles comme à la coopération entre les entreprises et les centres de recherche fondamentale (pilote: Forschungszentrum Jülich) ou appliquée (acteur central : Société Fraunhofer). Et elles se conjuguent à un intense effort de communication, via la création de pôles de compétence régionaux fédérateurs (des plateformes Internet) ou de centres dédiés, ainsi ce Centre de la pile à combustible (Zentrum für BrenstoffzellenTechnik, ZBT) qui vient d'être inauguré à Duisburg.

LE DYNAMISME DE L'INNOVATION ALLEMANDE EN MATIÈRE D'HYDROGÈNE est le fruit avant tout de l'activité industrielle et de son partenariat avec la recherche, qu'elle soit fondamentale ou appliquée. Ce n'est que récemment que les pouvoirs publics ont commencé à s'intéresser à ces technologies transversales, alertés par une industrie soucieuse de développer sa compétitivité, et conscients désormais de l'importance des technologies de l'hydrogène pour la politique générale d'innovation. Les préoccupations environnementales souvent mises en avant ne sont qu'une accroche thématique consensuelle placée, en réalité, au service d'activités industrielles dont on peut affirmer qu'elles sont très dynamiques outre-Rhin, bien qu'il soit impossible de les chiffrer. Transversales par définition, elles concernent en effet de nombreux domaines et compétences, dont ceux où l'économie allemande excelle : automobile, construction mécanique, chimie, engineering, science des matériaux...

De ce simple fait, le site Allemagne a un solide avantage compétitif. Si actuellement, l'hydrogène est encore l'affaire essentiellement des groupes les plus exposés à la compétition internationale, ceux-ci sont conscients que leur compétitivité est étroitement liée à celle de leurs réseaux de sous-traitants, c'est-à-dire à l'innovation des PME et à la qualification des artisans. Les relais de diffusion des savoirs sont dès lors aussi multiples que classiques : fédérations, presse professionnelle, universités, centres de recherche, salons professionnels, sites Internet. Mais il s'agit aussi de séduire les jeunes générations afin d'entretenir le goût des techniques comme des sciences de l'ingénieur dans l'opinion. Entreprises et pouvoirs publics ont ainsi développé un large éventail d'outils pédagogiques autour de la pile à combustible. Même le secteur du jeu éducatif s'y intéresse : on trouve dans le commerce un grand choix de kits d'expérimentation, et même cette " HyRunner » qui sera offerte aux équipes gagnantes du concours scolaire de Rhénanie du Nord-Westphalie. Parce qu'elles sont autrement consensuelles que les biotechnologies, les technologies de l'hydrogène ont un effet d'entraînement potentiel considérable sur la dynamique d'innovation générale de l'Allemagne.

\section{Indications bibliographiques}

COMMISSION EUROPÉENNE, "L'UE présente une vision pour la source d'énergie du XXlème siècle: I'hydrogène et la pile à combustible », IP/03/848, "'Feuille de route' de I'UE en vue d'un partenariat européen pour une économie durable de l'hydrogène ", IP/03/1229, « Un partenariat technologique européen fort pour progresser vers l'économie de l'hydrogène », IP/04/69 (www.europa.eu.int)

HyWays. The European Hydrogen Roadmap Project under FP6, Presented by U. Bünger, L-B-Systemtechnik, Bruxelles, 21-01-2004, (www.hynet.info)

JAN HOFMANN, "My home is my power plant. Mit Wasserstoff zur dezentralen Energieversorgung? ", Deutsche Bank Research, 02-12-2002

L-B-SYSTEMTECHNIK GMBH, Brennstoffzellen- und Wasserstoff-Technologien als wirtschaftliche Chance für Hamburg. Studie im Auftrag der Freien und Hansestadt Hamburg/Behörde für Umwelt und Gesundheit, janvier 2004 (www.fhh.hamburg.de)

Market Development of Alternative Fuels. Report of the Alternative Fuels Contact Group, décembre 2003 (www.hynet.info)

Sites Internet: www.brennstoffzelle.de; www.dwv-info.de; www.energieportal24.de ; www.hyweb.de ; www.initiative-brennstoffzelle.de ; www.ise-fhg.de ; www.kfa-juelich.de. 